1 ETVG/RUNX1 FUSION GENE ABROGATION DECREASES THE ONCOGENIC POTENCIAL

2 OF TUMOUR CELLS IN A PRECLINICAL MODEL OF ACUTE LYMPHOBLASTIC

\title{
3 LEUKAEMIA.
}

4

5 Adrián Montaño ${ }^{1}$, Jose Luis Ordoñez², Verónica Alonso-Pérez², Jesús Hernández-Sánchez ${ }^{1}$, Teresa

6 González ${ }^{3}$, Rocío Benito ${ }^{1}$, Ignacio García-Tuñón*², Jesús María Hernández-Rivas 1,2,3,4*

$8 \quad{ }^{1}$ IBSAL, IBMCC, Universidad de Salamanca-CSIC, Cancer Research Center; Salamanca; Spain.

$9 \quad{ }^{2}$ Unidad de Diagnóstico Molecular y Celular del Cáncer, Centro de Investigación del Cáncer-IBMCC

10 (USAL-CSIC), Salamanca, Spain.

$11{ }^{3}$ Dept of Hematology, Hospital Universitario de Salamanca; Salamanca; Spain.

$12{ }^{4}$ Dept of Medicine, Universidad de Salamanca, Spain.

13 *These authors share senior authorship.

Corresponding author:

16 Jesús-María Hernández-Rivas

17 IBMCC, CIC Universidad de Salamanca-CSIC, Hospital Universitario de Salamanca

$18 \quad$ Paseo de San Vicente 58

1937007 Salamanca

20 Spain

21 Phone: + 34923291384 // Fax: +34 923294624

22 e-mail: imhr@usal.es

24 Running title: $E / R$ maintains the oncogenic potential of ALL cells

25 Keywords

26 Acute lymphoblastic leukaemia, ETV6/RUNX1, CRISPR/Cas9, Genome edition. 


\section{ABSTRACT}

32 Background: The $\mathrm{t}(12 ; 21)(\mathrm{p} 13 ; \mathrm{q} 22)$, which fuses ETV6 and RUNX1 genes, is the most common genetic

33 abnormality in children with B-cell precursor acute lymphoblastic leukaemia. The implication of the

34 fusion protein in leukaemogenesis seems to be clear. However, its role in the maintenance of the disease

35 continues to be controversial. Aim: To eliminate the expression of the ETV6/RUNXI fusion gene, in order

36 to elucidate the effect in the leukaemic cells. Methods: Generation of an in vitro ETV6/RUNX1 knock out

37 model using the genetic modification system CRISPR/Cas9. Functional studies and generation of edited-

38 cell xenograft model were carried out. Results: For the first time, the expression of ETV6/RUNX1 fusion

39 gene was completely eliminated, thus generating a powerful model on which to study the role of the

40 fusion gene in leukaemic cells. ETV6/RUNX1 inactivation caused the deregulation of cellular processes

41 that could be participating in the maintenance of the leukaemic phenotype, such as differentiation and

42 lymphoid activation, apoptosis, cell signaling and cell migration. Tumour cells showed higher levels of

43 apoptosis, lower proliferation rate and a greater sensitivity to PI3K inhibitors in vitro along as a decrease

44 in tumour growth in xenografts models after ETV6/RUNX1 fusion gene abrogation. Conclusions:

45 ETV6/RUNX1 fusion protein plays a fundamental role in the maintenance of the leukaemic phenotype,

46 thereby being making the fusion protein a potential therapeutic target.

\section{BACKGROUND}

49 The gene fusion between the transcription factors ETV6 (TEL) and RUNXI (AMLI) is generated by $50 \mathrm{t}(12 ; 21)(\mathrm{p} 13 ; \mathrm{q} 22)$, the most frequent chromosomal translocation in children with acute lymphoblastic 51 leukaemia (ALL). ${ }^{1,2}$ Patients carrying this translocation are associated with a good prognosis and 52 excellent molecular response to treatment. However up to $20 \%$ of cases relapse. ${ }^{3-7}$ Furthermore, the 53 response to treatment of some relapse cases is associated with resistance to treatments such as 54 glucocorticoids (GCs), ${ }^{8}$ and these patients must be treated with stem cell transplantation. ${ }^{9}$

55 ETV6/RUNX1 (E/R) protein is known to play a role in the development of B-ALL, but by itself it is not 56 able of initiating the disease. Postnatal genetic events are required for the development of clinically overt 57 leukaemia. These second events are usually mutations or deletions, such as the loss of wild type (WT) 58 allele of ETV6. ${ }^{10}$ Recent studies suggest that $\mathrm{E} / \mathrm{R}$ is responsible for the initiation of leukaemia and also 59 essential for disease progression and maintenance, through deregulation of different molecular pathways 60 that contribute to leukaemogenesis. E/R regulates phosphoinositide 3-kinase (PI3K)/Akt/mammalian 
61 target of rapamycin (mTOR) (PI3K/AKT/mTOR) pathway, which promotes proliferation, cell adhesion

62 and DNA damage response; STAT3 pathway involved in self-renewal and cell survival and MDM2/TP53

63 whose deregulation induces the inhibition of apoptosis and consequently cell survival. ${ }^{11}$ Therefore, the

64 fusion protein could thus be intervening in relapse processes and in the lack of response to treatment.

65 However, the functional studies carried out by the silencing of $E / R$ fusion gene expression, mediated by 66 siRNA and shRNA, reveal that there is still controversy about the role of the oncoprotein in the 67 maintenance of the leukaemic phenotype. Thus E/R silencing by siRNA neither induced cell cycle 68 arrest/apoptosis nor attenuated clonogenic potential of cells. Therefore the E/R fusion protein may be 69 dispensable for the survival of definitive leukaemic cells. ${ }^{12}$ By contrast, other studies showed that E/R 70 expression was critical for the survival and propagation of the respective leukaemia cells in vitro and in 71 vivo. ${ }^{13}$ These results arise some doubts about the implications of the fusion protein in tumour cells.

72 The implementation of new genetic editing strategies has allowed the development of functional studies

73 by generation of gene and gene fusion $\mathrm{KO}$ models, both in vitro and in vivo. ${ }^{14}$ In this study, we 74 completely abrogated the expression of E/R fusion protein in REH ALL cell line using the CRISPR/Cas9 75 editing system and we studied the effects of genetic ablation of the fusion protein in cellular functions.

76 We also studied whether the suppression of E/R expression sensitize tumour cells to PI3K inhibitors.

77 Finally, we generated a xenograft mouse model to study the oncogenic potential of tumours cells after

78 E/R depletion. In summary, we provide evidence that fusion protein has a key role in the maintenance of 79 the leukaemic phenotype.

81 MATERIAL AND METHODS

\section{Cell lines and culture conditions}

83 REH, obtained from DMSZ German collection (ACC 22), is a cell line established from the peripheral 84 blood of a patient with ALL who carried $\mathrm{t}(12 ; 21)$ and $\operatorname{del}(12)$ producing respective $E / R$ fusion and 85 deletion of residual ETV6. REH was maintained in RPMI 1640 (Life Technologies, Carlsbad, California, 86 USA) supplemented with $15 \%$ fetal bovine serum (FBS) and $1 \%$ of Penicillin/ Streptomycin (P/S) (Life 87 Technologies). Stromal HS-5 cell line was obtained from ATCC collection (CRL-11882) and maintained 88 in DMEM (Life Technologies, Carlsbad, California, USA) supplemented with $10 \%$ FBS and $1 \%$ of P/S.

89 Both cells lines were maintained at $37^{\circ} \mathrm{C}$ with $5 \% \mathrm{CO} 2$.

$90 \quad$ sgRNAs design and cloning 
91 Based on the methodology of CRISPR/Cas9, two single guides RNAs (sgRNAs) (G1 and G2) were

92 designed with the Broad Institute CRISPR designs software (http://

93 www.broadinstitute.org/rnai/public/analysis-tools/sgrna- design). One of them directed towards the end of

94 exon 5 of ETV6 and other directed towards the beginning of intron 5-6, both before the fusion point, with

95 the intention of producing indels or deletions that modify the open reading frame of the oncogene, and,

96 therefore, the gene expression. These sgRNAs were cloned into a vector containing the Cas9 nuclease

97 coding sequence and GFP, pSpCas9(BB)-2A-GFP (PX458) (Addgene plasmid \#48138) ${ }^{15}$ as described

98 previously $^{14}$ (Supplementary Table 1 ). Then they were electroporated into the REH cells.

SgRNA transfections

100 REH ALL cells $\left(4 \times 10^{6}\right.$ cells) were electroporated with $4 \mu \mathrm{g}$ of both plasmid constructs ${ }^{14}(\mathrm{PX} 458 \mathrm{G} 1$

101 and PX458 G2) using the Amaxa electroporation system (Amaxa Biosystem, Gaithersburg, MD, USA)

102 according to supplier's protocol.

103 Flow cytometry analysis and cell sorting

104 Seventy-two hours after sgRNAs transfection, GFP-positive cells were selected by fluorescence-activated

105 cell sorting (FACS) using FACS Aria (BD Biosciences, San Jose, California, USA). Single-cells were

106 seeded in 96-well plate by FACS, establishing the different KO and control clones.

107 Sequencing of sgRNA targets sites

108 Genomic DNA was extracted using the QIAamp DNA Micro Kit (Qiagen, Hilden, Germany) following

109 the manufacturer's protocol. To amplify the region of $E / R$ fusion, PCR was performed using the

110 following primers: forward 5'- ACCCTCTGATCCTGAACCCC- $3{ }^{\prime}$ and reverse 5'-

111 GGATTTAGCCTCATCCAAGCAG- 3'. PCR products were purified using a High Pure PCR Product

112 Purification Kit (Roche, Basilea, Switzerland) and were sequenced by the Sanger method using each

113 forward and reverse PCR primers (Supplementary table 2).

114 The editing efficiency of the sgRNAs and the potential induced mutations were assessed using Tracking

115 of Indels by Decomposition (TIDE) software (https://tide-calculator.nki.nl; Netherlands Cancer Institute),

116 which only required two Sanger sequencing runs from wild-type cells and mutated cells.

117 Off-target sequence analysis

118 The top four predicted off-target sites obtained from "Breaking Cas" website

119 (http://bioinfogp.cnb.csic.es/tools/breakingcas/) were analyzed by PCR in the different clones

120 (Supplementary table 2) before to functional and xenograft experiments. 


\section{1 qPCR}

122 Total RNA extraction was performed with the RNeasy Kit (Qiagen) as suggested by the manufacturer.

123 Real-time reverse transcriptase- polymerase chain reactions (RT-PCRs) were performed as described ${ }^{16}$.

124 The primers for $E / R$ (sense, 5 - CTCTGTCTCCCCGCCTGAA

125 -3; antisense, 5 - CGGCTCGTGCTGGCAT-3), were designed. Real-time RT-PCR data shown include at

126 least 3 independent experiments with 3 replicates per experiment.

\section{Transcriptome sequencing}

128 RNA-seq was performed by using SMART-Seq v4 Ultra Low Input RNA kit (Clonthech, California,

129 U.S.). In all samples, RNA was analyzed following manufacturer's recommendations for the protocol

130 "Illumina library preparation using Covaris shearing". Libraries were sequenced in the HiSeq400

131 platform (Illumina) according to manufacturer's description with a read length of $2 \square \times \square 150$ nucleotides.

132 Briefly, bcl files were demultiplexing on BaseSpace (Illumina Cloud based resource) to generate fastq

133 files. Raw data quality control was performed with fastQc (v0.11.8), globin contamination was assessed

134 with HTSeq Count, FastQ screen evaluated ribosomal RNA contamination and other external possible

135 resources of contamination (Mus musculus, Drosophila melanogaster, Caenorhabditis elegans and

136 mycoplasma). STAR (v020201) was used for the alignment (hg19 reference genome) and FeatureCounts

137 (v1.4.6) to generate the read count matrix. Finally, DESeq2 was used for differentially gene expression

138 analysis. Of noted, DESeq2 model internally corrects for library size therefore normalizes the values and

139 enables paired comparisons. Heatmap was performed in R.

140 Go enrichment analysis (http://geneontology.org) to evaluate whether a set of genes was significantly

141 enriched between the different comparisons was used. The most significant biological mechanisms,

142 pathways and functional categories in the data sets of genes selected by statistical analysis were identified

143 through PANTHER Overrepresentation Test.

\section{Western blotting}

145 Protein expression was assessed by SDS-PAGE and western blotting (WB). The antibodies were obtained

146 from Cell Signaling Technology (Danvers, MA, USA) including a human anti-Bcl-2 antibody (1:1000;

147 2872) for Bcl-2, a human anti-Bcl-xL antibody (1:1000; 2762) for Bcl-xL, a human anti-phospho Akt

148 antibody $(1: 1000 ; 4060)$ for p-AKT (Ser473) and a human anti-phospho mTOR antibody $(1: 1000 ; 2971)$

149 for p-mTOR (Ser2448). Anti-rabbit IgG horseradish peroxidase-conjugated (1:5000; 7074) was used as a 
150 secondary antibody. Antibodies were detected using ECL ${ }^{\mathrm{TM}}$ WB Detection Reagents (RPN2209, GE

151 Healthcare, Illinois, Chicago, USA). ImageJ software was used for densitometric analysis. ${ }^{17,18}$

152 Apoptosis, cell cycle analysis and proliferation assays

153 Apoptosis was measured by flow cytometry with an annexin V-Dy634 apoptosis detection kit

154 (ANXVVKDY, Immunostep, Salamanca, Spain) following the manufacturer's instructions. Briefly, $5 \times$

$15510^{5}$ cells were collected and washed twice in PBS and labeled with annexin V-DY-634 and non-vital dye

156 propidium iodide (PI), allowing the discrimination of living-intact cells (annexin-negative, PI-negative),

157 early apoptotic cells (annexin-positive, PI-negative) and late apoptotic or necrotic cells (annexin-positive,

158 PI-positive). In parallel, cell distribution in the cell cycle phase was also analyzed by measuring DNA

159 content (PI labeling after cell permeabilization). These experiments were carried out after 24, 48 and 72

160 culture hours.

161 For proliferation measuring, MTT assays and labeling of cells with CellTrace CFSE Cell Proliferation Kit

162 (Thermo Fisher) were used. In MTT assays, cells were plated on 96-well plates, cell density varied

163 according to the days of the experiment, in a range between $3 \times 10^{4}$ and $5 \times 10^{3}$ cells (24-240 hours). MTT

164 solution (3-(4,5-cimethylthiazol-2-yl)-2,5-diphenyl tetrazolium bromide) was added at concentration of

$1650.5 \mu \mathrm{g} / \mu \mathrm{L}$ (Merck, Darmstadt, Germany). After incubation for 3-4 hours at $37^{\circ} \mathrm{C}$, cells were lysed with the

166 solubilization solution $(10 \% \mathrm{SDS}$ in $0.01 \mathrm{M} \mathrm{HCl})$ and absorbance was measured in a plate reader at 570

$167 \mathrm{~nm}$. For labeling of cells, $3 \square \times \square 10^{5}$ cells were stained with CellTrace-CFSE following the manufacturer's

168 instructions and plated on 6-well plates. After 48 hours, CFSE expression was measured by flow

169 cytometry.

\section{B-ALL-stromal cell co-culture}

171 HS-5 human mesenchymal stromal cells (MSCs) were plated at a density of $1 \square \times \square 10^{5}$ cells per coverslip 172 in 6-well plates. After $24 \square \mathrm{h}$, control cells and E/R KO clones were stained with Celltrace-CFSE and

$1733 \square \times \square 10^{5}$ cells placed on top of the stromal cell monolayer. Cells were co-cultured during 48h in RPMI

1741640 supplemented with $15 \%$ FBS and $1 \%$ of $\mathrm{P} / \mathrm{S}$ at $37^{\circ} \mathrm{C}$ with $5 \% \mathrm{CO} 2$.

\section{Drugs and treatments}

176 The followings drugs were used: Vincristine and Copanlisib (BAY 80-6946) were obtained from

177 Selleckchem (Houston, USA) and Prednisolone (P6004) obtained from Merck. All drugs were prepared at

178 the appropriate stocking concentrations in DMSO (Merck) and stored at $-20^{\circ} \mathrm{C}$ until use.

\section{Mouse xenograft tumourigenesis}


18016 four- to five-week-old female NOD/SCID/IL2 receptor gamma chain null (NSG) mice (Charles River,

181 Barcelona, Spain) were used. $5 \times 10^{6}$ tumour cells from REH or control clone were subcutaneously

182 injected into the left flank and tumour cells from $\mathrm{KO}$ clones (KO1, $\mathrm{KO} 2$ and $\mathrm{KO} 3$ ) were injected in the

183 right flank as described previously. ${ }^{14} \mathrm{REH}$ vs $\mathrm{KO} 2$ in the group 1, REH vs KO3 in the group 2, control

184 clone vs KO1 in the group 3 and control clone vs KO2 in the group 4 (4 mice per group). The study

185 received prior approval from the Bioethics Committee of our institution and followed the Spanish and

186 European Union guidelines for animal experimentation (RD 53/2013 and 2010/63/UE).

187 Tumour diameters were measured every 2-3 days with a caliper. Tumour volume was calculated as

188 described elsewhere by the formula $a 2 b \pi / 6$ ( $a$ and $b$ being, respectively, the smallest and the biggest

189 diameters). Mice were sacrificed by anesthesia overdose when tumour volume reached $2 \mathrm{~cm}^{3}$ or $48-62$

190 days after cell injection, upon which the tumours were collected and weighted.

\section{Histopathology and immunohistochemistry}

192 Excised tumours were sampled just after sacrifice and representative areas were a) formalin-fixed (24

193 hours) (Merck Millipore) and paraffin-embedded and (b) snap-frozen in OCT and stored at $80^{\circ} \mathrm{C}$ as

194 previously described. ${ }^{19}$ Tissue sections $2 \mu \mathrm{M}$ thick were stained with hematoxilin \& eosin (H\&E) and

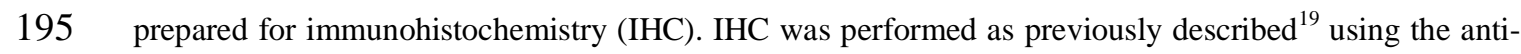

196 Ki67primary antibody (Merck Millipore). The number of mitotic figures were counted in 6 high-power

197 field.

\section{Statistical analysis}

199 Statistical analysis was performed using GraphPad Prism 6 Software. Differences in relative expression

200 of E/R and cell viability after treatments were tested by Tukey's multiple comparisons test. Differences in

201 protein expression, proliferation and apoptosis levels were tested by unpaired $t$-test. Differences in

202 tumour masses over time were tested by non-parametric Mann Whitney U test followed by Tukey’s

203 multiple comparisons test and parametric Student's t test. Statistical significance at values of $P \leq 0,05(*)$,

$204 P \leq 0.005(* *)$ and $P \leq 0,001(* * *)$ was noted.

\section{RESULTS}

207 CRISPR/Cas9 edited lymphoid cell line showed a loss of E/R functionality

$208 E / R$ sequence was edited by CRISPR/Cas9 and evaluated by Sanger sequencing in REH cells

209 (Supplementary Figure 1). The edition efficiency evaluated through TIDE was 62,6\% with sgRNA G1 
210 and 70,6\% with sgRNA G2. The most frequent generated mutations were insertions up to 4 base pairs

211 (bps).

212 Single-edited cells were seeded into a 96 well plate to obtain clones with a single edition that predicted a

213 KO sequence for the oncogene. Only 48 single cell clones proliferated in culture. These clones were

214 screened by sanger sequencing and the results revealed that more than $50 \%$ of clones (25/48) presented an

215 edited $E / R$ sequence. Among them, three single-edited cell clones "KO1, KO2 and KO3 clones" with a

216 predicted $E / R$ KO sequence were selected for the study. These clones had different editions in their

217 sequences. KO1 clone carried an insertion of two cytosines at the end of exon 5, near the PAM sequence

218 of the $\operatorname{sgRNA~G2,~thus~a~frameshift~mutation~that~generated~a~stop~codon~before~finishing~the~exon.~On~}$

219 the other hand, $\mathrm{KO} 2$ and $\mathrm{KO} 3$ had an insertion of 5 and 3 nucleotides respectively near the PAM

220 sequence within exon 5, followed by a deletion of 100 bps approximately between both sgRNAs. These

221 alterations modified the open reading frame, generating the stop codon in the next exon. In addition, the

222 loss of the splicing region prevented the correct processing of the protein. Additionally, two single-edited

223 cell clones with WT E/R sequence were used as control clones "Control 1 and Control 2" (Supplementary

224 Figure 2).

225 In order to check the functionality of the $E / R$ alleles carrying these clones, the expression of the fusion

226 transcript E/R was quantified by quantitative PCR. For that, RNAs of the different clones were extracted

227 and transcribed to cDNA. Quantification revealed a total loss of E/R mRNA expression in KO2 and KO3

228 clones as compared to control clones $(P<0.001)$ and a leaky expression in KO1 clone $(P<0.001)($ Figure

229 1).

230 The four most likely off-target sequences from both guides were analyzed by sequencing Sanger into the

231 different clones. The study of the obtained sequences revealed the lack of editing in those regions,

232 confirming the absence of CRISPR/Cas9 off-targets (data not shown).

233 Transcriptomic analysis of E/R KO lymphoid cell line generated by CRISPR/Cas9 showed a

234 distinct expression signature and a deregulation of its downstream signaling genes

235 The gene expression profile analysed by total RNA-sequencing showed a distinct expression signature in

236 E/R KO clones as compared with REH cells and control clones. 342 genes were significantly deregulated

237 after $E / R$ fusion gene depletion (q<0.05), 180 upregulated and 160 downregulated (Supplementary table

238 3). Some of these genes have been previously associated with ALL pathology and have also been shown 
239 to be directly regulated by $R U N X 1$ transcript factor ${ }^{20}$ (Table 1$)$. The gene expression profile of the top50

240 of the most deregulated genes according to fold change (FC) values is shown in Figure 2.

241 In order to elucidate the effect of $E / R$ fusion gene abrogation on a functional level, the significantly

242 deregulated genes were grouped into a cellular processes according to their function by enrichment

243 analysis (Supplementary table 4). These cellular processes were manually classified into 11 categories:

244 differentiation and lymphoid activation, immune response, response to stimuli, cell activation, apoptosis,

245 cell signaling, chemical homeostasis, GPTase regulation, cell migration, location of cellular components

246 and neuronal system development.

247 Apoptosis was one of the altered cellular processes. In particular, an overexpression of miR-146 was

248 observed in the $\mathrm{E} / \mathrm{R} \mathrm{KO}$ clones as compared with control clones $(P<0.001)$. miR-146 can regulate the

249 expression of the apoptosis factor STATI, and the anti-apoptosis factor $B c l-x L$, thus promoting the

250 apoptosis of ALL cells. ${ }^{21}$ Furthermore, tumour protein P63 gene (TP63 gene) was also upregulated in E/R

251 KO clones as compared with control clones $(P<0.001)$. This gene is involved in an antiapoptotic pathway

252 that regulates the normal survival of B cells. ${ }^{22,23}$

253 An overexpression of RGS16 was also observed in ALL cells after $E / R$ abrogation. This gene plays an 254 antiproliferative role through inhibition of the PI3K/AKT/mTOR pathway ${ }^{24-26}$ and inhibition of cell 255 migration. ${ }^{27}$ On the other hand, the tumour suppressor PTPKR whose expression prevents the activation 256 of pathways such as PI3K/AKT/mTOR and STAT signaling pathways ${ }^{28}$ was downregulated after $E / R$ 257 fusion gene abrogation. This gene was found downregulated in some types of cancer such as ovarian, 258 breast and NK-T cell lymphoma, ${ }^{29-31}$ but also in ALL cell lines. ${ }^{28}$ However, E/R positive ALL patients 259 have shown an overexpression of PTPKR. ${ }^{32,33}$

\begin{tabular}{|c|c|c|c|}
\hline Gene & $\begin{array}{c}\text { Overexpressed/ } \\
\text { Infraexpressed } \\
(\text { E/R KO) }\end{array}$ & Function & ALL patients \\
\hline CXCR7 & Infraexpressed & Cell proliferation and migration ${ }^{34-39}$ & Overexpressed ${ }^{40}$ \\
\hline$L C K$ & Infraexpressed & $\begin{array}{l}\text { Lymphoid activation and development, GC } \\
\text { resistance }{ }^{41-43}\end{array}$ & $\begin{array}{l}\text { Overexpressed in patients with } \\
\text { prednisone poor response }{ }^{41}\end{array}$ \\
\hline
\end{tabular}




\begin{tabular}{|c|c|c|c|}
\hline$P T P R G$ & Infraexpressed & $\begin{array}{l}\text { Cell growth, differentiation, mitotic cycle and } \\
\text { oncogenic transformation }^{44,45}\end{array}$ & $\begin{array}{l}\text { Infraexpressed in in high } \\
\text { hyperdiploid childhood }^{46}\end{array}$ \\
\hline PIK3C3 & Infraexpressed & $\begin{array}{l}\text { Regulation of autophagy }{ }^{47} \text { Protection of leukaemic } \\
\text { cells during chemotherapeutic treatment }{ }^{48}\end{array}$ & $\begin{array}{l}\text { Overexpressed in E/R positive } \\
\text { patients } \\
\text { 32,48 }\end{array}$ \\
\hline PTPRK & Infraexpressed & Tumour suppressor ${ }^{28}$ & $\begin{array}{l}\text { Overexpression in } \mathrm{E} / \mathrm{R} \text { positive } \\
\text { patients } \mathrm{s}^{32,33}\end{array}$ \\
\hline$m i R-146$ & Overexpressed & Innate immunity ${ }^{49}$ and apoptosis ${ }^{21}$ & Overexpression in children ${ }^{50}$ \\
\hline$R X R A$ & Overexpressed & $\begin{array}{l}\text { Retinoic acid X receptor that serves as a therapeutic } \\
\qquad \operatorname{target}^{51}\end{array}$ & $\begin{array}{l}\text { Overexpressed in patients with } \\
\qquad I K Z F 1 \text { alterations }{ }^{51}\end{array}$ \\
\hline$R G S 16$ & Overexpressed & $\begin{array}{l}\text { Antiproliferative role }{ }^{24-26} \text { and inhibition of cell } \\
\text { migration }^{27}\end{array}$ & $\begin{array}{l}\text { Overexpression in high hyperdiploid } \\
\text { childhood }^{52}\end{array}$ \\
\hline$T L R 7$ & Overexpressed & Immune system ${ }^{53}$ & Infraexpressed ${ }^{54,55}$ \\
\hline
\end{tabular}

267 To elucidate the biological effects of abrogation of E/R expression in the KO clones, several studies were performed. MTT proliferation studies were performed at 24-hour intervals up to 240 hours. The results showed no proliferation differences between KO clones and REH cells or control clones (Supplementary Figure 3A). We simultaneously analyzed the cell cycle distribution of the different cells by permeabilization followed by PI staining. No differences were observed between the different clones (Figure 3A). No significant differences were observed through the expression of CFSE by flow cytometry

273 (Figure 3B). However, E/R KO clones showed a significantly lower proliferation rate than control clones when they were co-cultured with MSCs (HS-5) $(P<0.05)$ (Figure 3C).

275 Deregulation of genes such as miR-146a or TP63 observed by expression analysis suggested the alteration 276 of cellular processes such as the regulation of apoptosis. Levels of anti-apoptotic factor such us $B c l-2$ or $B c l-x L$ gene have shown to play a key role in the survival of $\mathrm{E} / \mathrm{R}$ positive cells, protecting from 278 programmed death. ${ }^{56,57}$ To check these findings, Bcl-2 and Bcl-xL expression levels were measured through WB. Suppression of the fusion protein produced a decrease of $60 \%$ and $47 \%$ in the expression of

$280 \mathrm{Bcl}-2$ and Bcl-xL proteins respectively $(P=0.003 ; P=0.043)$, thus reducing the resistance to apoptosis provided by the antiapoptotic factors of this family (Figure 4). In agreement with this observation, we 282 detected an increased in the late apoptotic levels assessed by annexin $\mathrm{V}$ and propidium iodide staining in 283 E/R KO clones as compared with control clones $(8.99 \pm 2.08$ vs $2.135 \pm 0.065)(P<0.05)$ (Figure 3D). 
285 by induction of apoptosis in mitotic cells, ${ }^{58}$ induced a greater late apoptotic rate in E/R KO clones as

286 compared with control clones $(75.8 \pm 9.59$ vs $32.7 \pm 2.1)(P<0.05)$ (Figure $3 \mathrm{E})$.

287 Abrogation of ETV6/RUNX1 expression enhances sensitivity to the PI3K inhibitor Copanlisib

288 Deregulation of RGS16 or PTPKR genes also suggested the alteration of the PI3K/AKT/mTOR pathway.

289 Several studies have already suggested that E/R may be key in the maintenance of the leukaemic

290 phenotype through the activation of different pathways, including the PI3K/AKT/mTOR pathway, ${ }^{13,59}$

291 resulting in proliferation and cell survival of leukaemic cells. Akt phosphorylation levels measured

292 through WB showed a reduction of $90 \%$ in Akt activity in the KO clones relative to REH cells and

293 control clones $(P=0.003)$, suggesting the decrease in PI3K/Akt/mTOR activity as a result of the

294 elimination of the expression of E/R (Figure 4).

295 A large proportion of relapsing positive E/R patients become resistant to GCs such as Prednisolone,

296 widely used in ALL treatment. ${ }^{8}$ Fuka's group demonstrated that the use of PI3K inhibitors can sensitize

297 positive E/R cells to GCs. ${ }^{13}$

298 After verifying a lower activation levels of PI3K/Akt/mTOR pathway with the elimination of E/R

299 expression, we aimed to test if these cells responded in the same way to PI3K inhibitors. For that, we

300 used Copanlisib, a PI3K inhibitor with inhibitory activity predominantly against the PI3K-alpha and

301 PI3K-delta isoforms. ${ }^{60,61}$ Treatment with Copanlisib $(10 \mathrm{mM})$ resulted in higher decrease of viability in

302 E/R KO clones compared with REH cells and controls clones (Figure 5A). To verify if Copanlisib was

303 actually inhibiting the PI3K/Akt/mTOR pathway, we measured the phosphorylation levels of Akt and

304 mTOR by WB, before and after treatment. We observed that the phosphorylation levels of both proteins

305 decreased after treatment (Figure 5B).

306 On the other hand, treatment with Prednisolone $(250 \mu \mathrm{M})$ was comparable to the effect of Copanlisib on

307 E/R positive cells. We did not observe a higher decrease of cell viability in E/R KO clones as compared

308 with REH cells and control clones (Figure 5C). A joint exposure of Copanlisib (10 nM) and Prednisolone

$309(250 \mu \mathrm{M})$ showed a decrease of cell viability in REH and control clones expressing $E / R$ fusion gene as

310 compared with Prednisolone and Copanlisib alone. Furthermore, we also observed greater reduction of

311 cell viability in E/R KO clones as compared with REH cells and control clones (Figure 5C).

\section{E/R repression impairs the tumourigenic capacity in vivo}

313 In order to determine the effects of E/R expression abrogation in vivo, 16 NSG mice were subcutaneously

314 injected with REH cells or control clone (left flank) and KO clones (right flank). Only 6 mice injected 
315 with KO clones developed tumour growth on the right flank (6/16), whereas all those injected with REH

316 or control clone developed a tumour (16/16). In the first group (REH cells vs KO2 clone), none of flanks

317 injected with KO2 developed tumour (mean mass: $0 \mathrm{mg} \pm 0$ vs $4872.5 \mathrm{mg} \pm 1323 ; P=0.029$ ). In the

318 second group (REH cells vs KO3 clone), only one of flanks injected with KO3 developed a tumour (1/4).

319 This tumour was significantly smaller than those generated from REH cells (mean mass: $40 \mathrm{mg} \pm 69.3 \mathrm{vs}$

$3204212.5 \mathrm{mg} \pm 1663.9 ; P=0.029)$. In the third group (control clone vs KO1), we observed tumour growth in

$3212 / 4$ flanks of mice injected with KO1. These tumours were significantly smaller than those generated

322 from control clone (mean mass: $483 \mathrm{mg} \pm 354.4$ vs $2470 \mathrm{mg} \pm 872.5$ vs $P=0.041$ ). In the same way, $3 / 4$

323 mice develop tumours from $\mathrm{KO} 2$ in the group 4 (control clone vs $\mathrm{KO} 2$ ), but these tumours were

324 significantly smaller than those generated from control clone (mean mass: $355 \mathrm{mg} \pm 293.6 \mathrm{vs} 2255 \mathrm{mg}$

$325 \pm 1215.6 ; P=0.029$ ) (Figure $6 \mathrm{~A}$ and Supplementary figure 4). In general, subcutaneous tumours

326 generated from E/R KO cells were significantly smaller than those produced by REH cells or control

327 clone (mean mass: $202 \mathrm{mg} \pm 298.9$ vs $4542.5 \mathrm{mg} \pm 1539 ; P<0.001 / /$ vs $2347.1 \mathrm{mg} \pm 1087.2 ; P \leq 0.001)$

328 (Figure 6B).

329 In addition, significant differences were observed in the time of appearance of the tumours. Those

330 tumours generated through the KO clones appeared around day 42 (mean: $46.5 \pm 7.8$ ), unlike those

331 generated by the REH cells or the control clone, which appeared around day 29 (mean: $29 \pm 4.2$;

$332 P=0.001$ ) and day 36 (mean: $36.6 \pm 5.34 ; P=0.03$ ) respectively (Figure 6C).

333 Histopathological analysis of representative tumours from each group of mice revealed a higher number

334 of mitotic figures in tumours from REH (52 vs $20, P=0.017)$ and control clones (62 vs $20 ; P=0.006)$ as

335 compared to tumours from KOs clones. In KOs tumours, but not in REH and control clone tumours, we

336 also observed the "starry sky" (macrophages containing dead apoptotic tumour cells) (Supplementary

337 figure 5). No other morphological changes between tumours were observed.

\section{DISCUSSION}

340 In this study, we generated an E/R KO model in an ALL cell line carrying the $\mathrm{t}(12 ; 21)$ in order to assess

341 how the loss of fusion expression affects the tumour cells. This translocation is the most common in

342 children with $\mathrm{ALL}^{1,2}$ and encodes a chimeric transcription factor that converts $R U N X 1$ from a

343 transcriptional modulator to a transcriptional repressor of $R U N X 1$ target genes. ${ }^{62}$ The expression of E/R

344 results in the generation of a persistent pre-leukaemic clone, which requires of secondary genetic 
345 abnormalities to converts to ALL. ${ }^{57,63}$ However, the implication of the E/R expression in the maintenance

346 of the disease is not quite clear. Previous studies have already silenced the fusion protein by using RNAi

347 and shRNA, but they did not completely eliminate the levels of the protein. In addition, these studies

348 showed contradictory results, which suggest that more studies are needed to help elucidate the true

349 oncogenic potential of the fusion protein. ${ }^{12,13}$

350 The recent evolution of genetic editing techniques with the CRISPR/Cas9 system has allowed, among

351 others, the generation of KO gene models, helping us to better understanding the biology of diseases such

352 as ALL. ${ }^{64}$ We used CRISPR/Cas9 system to completely eliminate the expression of the E/R fusion

353 protein. The loss of fusion expression was checked, observing an absence of mRNA coding for E/R in the

$354 \mathrm{KO} 2$ and $\mathrm{KO} 3$ clones and a leaky expression in $\mathrm{KO} 1$ clone. We hypothesize that this loss of mRNA was

355 due to nonsense-mediated mRNA decay mechanism. ${ }^{65}$ In this way we generated a powerful model on

356 which to study the effect of the elimination of the fusion gene on leukaemic cells. These cells maintain a

357 series of secondary alterations that triggered the leukaemia, similar as occurs in patients.

358 Transcriptome analysis of different clones showed a huge number of genes significantly deregulated after

$359 E / R$ abrogation. A greater number of upregulated genes was observed which agrees with the repressive

360 activity of the $E / R$ fusion gene. ${ }^{62,66}$ The most of genes significantly deregulated are involved in different

361 cellular processes such us differentiation and lymphoid activation, apoptosis, cell signaling and cell

362 migration. These results are in agreement with previous studies, in which was demonstrated the

363 implication of $\mathrm{E} / \mathrm{R}$ in the cellular processes that may be maintaining the leukaemic state of the tumour

364 cells. $^{13,57,59,66}$ The expression of some of these genes has been described as a specific signature of E/R

365 positive ALL patients. ${ }^{32,33}$

366 Within of the top50 of the significantly deregulated genes in our study we observed a series of

367 downregulated genes after $E / R$ abrogation such as $C X C R 7, L C K$ and PTPGR and other upregulated genes

368 such as Mir-146, RXRA, RGS16, TP63 and IL7R. The reverse deregulation of these genes, often seen in

369 ALL patients, leads to increased migratory, tumour activation or chemotherapy resistance effects.

370 Very subtle changes were observed in cell proliferation and cell cycle distribution after $E / R$ abrogation in

371 vitro, in agreement with previous studies. ${ }^{12,13}$ However, E/R KO clones showed a significantly lower

372 proliferation rate when they were co-cultured with MSC. Mesenchymal cells have been shown to play a

373 key role in the development and evolution of $\mathrm{ALL}^{67,68}$ and Bonilla et al. (2019) observed in a recently

374 study that MSCs induce greater cell adhesion, higher proliferation ratio and greater migration capacity to 
375 REH cells. ${ }^{69}$ Our data show that the $E / R$ fusion gene therefore participates in the interaction of leukaemic

376 cells with the microenvironment and the loss of $E / R$ fusion gene expression reverses the proliferative

377 capacity that MSCs confer on leukaemia cells. E/R KO clones also showed a higher late apoptosis rate,

378 demonstrating that the fusion gene regulates the expression of antiapoptotic factors that protect leukaemic

379 cells from apoptosis. Death levels were also higher in E/R KO clones after treatment with Vincristine.

380 On the other hand, we wanted to check if the non-expression of $E / R$ and consequently the loss of 381 activation of the PI3K/Akt/mTOR pathway, was able to sensitize the cells to PI3K inhibitors. The use of 382 PI3K inhibitors alone has shown to be an effective treatment in E/R positive cells. Furthermore, the 383 activity of these inhibitors in combination with Prednisolone, a GC widely used in the treatment of ALL,

384 has been shown to decrease the resistance offered by positive E/R cells to GCs. In our study, we observed 385 that use of Copanlisib, a PI3K inhibitor, achieved a significantly decrease of cell viability in E/R KO 386 clones as compared with E/R positive cells. We also observed that treatment with Copanlisib achieved the 387 sensitization to Prednisolone in E/R positive cells as Fuka's study described. ${ }^{13}$ However, in our study, this 388 sensitization was even greater in E/R KO cells. Therefore, our data demonstrate that the fusion gene may

389 be a good therapeutic target with which to improve the drug sensitivity of positive E/R cells.

390 Finally, we wanted to check if E/R abrogation also decreased the tumour potential of cells in vivo. For 391 that, a xenograft model was generated by injecting these cells into immunosuppressed mice, taking the 392 injection of REH cells or a control clone on the opposite flank as control. Mice injected with KO clone

393 cells did not generated tumours or generated smaller tumours than those generated by REH cells or 394 control clone. The higher rate of mitotic activity in REH and control tumours observed through the 395 histopathology analysis explains the greater growth of these tumours and reveals a greater tumoural 396 capacity of these cells carrying $E / R$ fusion gen in vivo.

397 Together these data suggest that the $E / R$ fusion gene has a key role in the maintenance of the leukaemic 398 phenotype. By eliminating E/R expression, the cells lost tumourigenic capacity, becoming more sensitive 399 to drugs and reducing their oncogenic capacity in vitro and in vivo. Therefore, although more studies are 400 needed to elucidate the mechanism of action of the fusion gene, this study demonstrates that it could be a 401 possible therapeutic target to design new drugs that prevent the correct expression of this protein. 
404 AM, IGT, RB and JMHR designed research. AM performed the experiments, compiled data and drafted

405 the manuscript. JLO was responsible for mouse experiments and drug tests. JHS was responsible for 406 sequencing experiments. VAP carried out additional experimental work. IGT, RB, JMHR, JLO, VAP and

407 TG helped interpret the results and write the manuscript. AM wrote the paper with input from all authors.

\section{ACKNOWLEDGEMENTS}

410 We thank Sara González, Irene Rodríguez, Maribel Forero-Castro, Ana Marín-Quílez, María Herrero-

411 García, Almudena Martín-Martín, Sandra Santos, Cristina Miguel, from the Cancer Research Center of 412 Salamanca, Spain. We are grateful to Ángel Prieto and Ana I García, María Luz Sánchez and María

413 Carmen Macías from the Microscopy Unit, Cytometry Unit and Molecular Pathology Unit, respectively,

414 from the Cancer Research Center of Salamanca for the technical assistance. We thank Luis Muñoz and all 415 the members from the Animal Experimentation Research Center from the University of Salamanca. We 416 also thank Mercedes Garayoa for providing us with the HS-5 cell line.

\section{FUNDING}

419 This work was financially supported in part by a grant from the Consejería de Educación, Junta de 420 Castilla y León, Fondos FEDER (SA085U16, SA271P18), and the Regional Council of Castilla y León 421 SACYL, (GRS 1847/A/18), Fundación Castellano Leonesa de Hematología y Hemoterapia (FUCALHH 422 2017), Proyectos de investigación en Biomedicina, gestión sanitaria y atención sociosanitaria del IBSAL 423 (IBY17/00006), Fundación Memoria Don Samuel Solórzano Barruso, Centro de Investigación Biomédica 424 en Red de Cáncer (CIBERONC CB16/12/00233), a grant to JLO from the University of Salamanca 425 ("Contrato postdoctoral programa II 2017-18”), and a grant to AM from the Junta Provincial de 426 Salamanca of the Asociación Española Contra el Cáncer (AECC).

\section{COMPETING INTERESTS}

429 The authors declare that they have no competing interests

\section{REFERENCES}


4321 Pui, C. H., Relling, M. V. \& Downing, J. R. Acute lymphoblastic leukemia. The

$433 \quad$ New England journal of medicine 350, 1535-1548, doi:10.1056/NEJMra023001

434 (2004).

4352 Shurtleff, S. A. et al. TEL/AML1 fusion resulting from a cryptic $t(12 ; 21)$ is the

436

437

438

439

440

441

442

443

444

445

446

447

448

449

450

451

452

453

454

455

456

457

458

459

460

461

462

463

464

465

466

467

468

469

470

471

472

473

474

475

476

477

478

479 most common genetic lesion in pediatric ALL and defines a subgroup of patients with an excellent prognosis. Leukemia 9, 1985-1989 (1995).

3 Conter, V. et al. Molecular response to treatment redefines all prognostic factors in children and adolescents with B-cell precursor acute lymphoblastic leukemia: results in 3184 patients of the AIEOP-BFM ALL 2000 study. Blood 115, 32063214, doi:10.1182/blood-2009-10-248146 (2010).

4 Moorman, A. V. et al. Prognostic effect of chromosomal abnormalities in childhood B-cell precursor acute lymphoblastic leukaemia: results from the UK Medical Research Council ALL97/99 randomised trial. The lancet oncology 11, 429-438, doi:10.1016/S1470-2045(10)70066-8 (2010).

5 Pui, C.-H., Robison, L. L. \& Look, A. T. Acute lymphoblastic leukaemia. The Lancet 371, 1030-1043, doi:10.1016/s0140-6736(08)60457-2 (2008).

6 Rubnitz, J. E., Downing, J. R. \& Pui, C. H. Significance of the TEL-AML fusion gene in childhood AML. Leukemia 13, 1470-1471 (1999).

7 Uckun, F. M. et al. Expression of TEL-AML1 fusion transcripts and response to induction therapy in standard risk acute lymphoblastic leukemia. Leuk Lymphoma 42, 41-56, doi:10.3109/10428190109097675 (2001).

8 Bokemeyer, A. et al. Copy number genome alterations are associated with treatment response and outcome in relapsed childhood ETV6/RUNX1-positive acute lymphoblastic leukemia. Haematologica 99, 706-714, doi:10.3324/haematol.2012.072470 (2014).

9 Kuster, L. et al. ETV6/RUNX1-positive relapses evolve from an ancestral clone and frequently acquire deletions of genes implicated in glucocorticoid signaling. Blood 117, 2658-2667, doi:10.1182/blood-2010-03-275347 (2011).

10 Raynaud, S. et al. The 12;21 translocation involving TEL and deletion of the other TEL allele: two frequently associated alterations found in childhood acute lymphoblastic leukemia. Blood 87, 2891-2899 (1996).

11 Sun, C., Chang, L. \& Zhu, X. Pathogenesis of ETV6/RUNX1-positive childhood acute lymphoblastic leukemia and mechanisms underlying its relapse. Oncotarget 8, 35445-35459, doi:10.18632/oncotarget.16367 (2017).

12 Zaliova, M., Madzo, J., Cario, G. \& Trka, J. Revealing the role of TEL/AML1 for leukemic cell survival by RNAi-mediated silencing. Leukemia 25, 313-320, doi:10.1038/leu.2010.277 (2011).

13 Fuka, G. et al. Silencing of ETV6/RUNX1 abrogates PI3K/AKT/mTOR signaling and impairs reconstitution of leukemia in xenografts. Leukemia 26, 927-933, doi:10.1038/leu.2011.322 (2012).

14 Garcia-Tunon, I. et al. The CRISPR/Cas9 system efficiently reverts the tumorigenic ability of BCR/ABL in vitro and in a xenograft model of chronic myeloid leukemia. Oncotarget 8, 26027-26040, doi:10.18632/oncotarget.15215 (2017).

15 Ran, F. A. et al. Genome engineering using the CRISPR-Cas9 system. Nat Protoc 8, 2281-2308, doi:10.1038/nprot.2013.143 (2013).

16 Martinez, N. et al. The oncogenic fusion protein RUNX1-CBFA2T1 supports proliferation and inhibits senescence in $\mathrm{t}(8 ; 21)$-positive leukaemic cells. $B M C$ Cancer 4, 44, doi:10.1186/1471-2407-4-44 (2004). 
48117 Abramoff, M. D., Magalhães, P. J. \& Ram, S. J. Image Processing with ImageJ. FLI1, and SCL binding in megakaryocytes identifies hematopoietic regulators. Developmental cell 20, 597-609, doi:10.1016/j.devcel.2011.04.008 (2011).

21 Yan, W. et al. The effect of miR-146a on STAT1 expression and apoptosis in acute lymphoblastic leukemia Jurkat cells. Oncology letters 13, 151-154, doi:10.3892/ol.2016.5395 (2017).

22 Binsky, I. et al. TAp63 regulates VLA-4 expression and chronic lymphocytic leukemia cell migration to the bone marrow in a CD74-dependent manner. $J$ Immunol 184, 4761-4769, doi:10.4049/jimmunol.0904149 (2010).

23 Lantner, F. et al. CD74 induces TAp63 expression leading to B-cell survival. Blood 110, 4303-4311, doi:10.1182/blood-2007-04-087486 (2007).

24 Liang, G., Bansal, G., Xie, Z. \& Druey, K. M. RGS16 inhibits breast cancer cell growth by mitigating phosphatidylinositol 3-kinase signaling. The Journal of biological chemistry 284, 21719-21727, doi:10.1074/jbc.M109.028407 (2009).

25 Liu, T. et al. The retinoid anticancer signal: mechanisms of target gene regulation. British journal of cancer 93, 310-318, doi:10.1038/sj.bjc.6602700 (2005).

26 Vasilatos, S. N. et al. Crosstalk between lysine-specific demethylase 1 (LSD1) and histone deacetylases mediates antineoplastic efficacy of HDAC inhibitors in human breast cancer cells. Carcinogenesis 34, 1196-1207, doi:10.1093/carcin/bgt033 (2013).

27 Berthebaud, M. et al. RGS16 is a negative regulator of SDF-1-CXCR4 signaling in megakaryocytes. Blood 106, 2962-2968, doi:10.1182/blood-2005-02-0526 (2005).

28 Stevenson, W. S. et al. DNA methylation of membrane-bound tyrosine phosphatase genes in acute lymphoblastic leukaemia. Leukemia 28, 787-793, doi:10.1038/leu.2013.270 (2014).

29 Sun, P. H., Ye, L., Mason, M. D. \& Jiang, W. G. Protein tyrosine phosphatase kappa (PTPRK) is a negative regulator of adhesion and invasion of breast cancer cells, and associates with poor prognosis of breast cancer. J Cancer Res Clin Oncol 139, 1129-1139, doi:10.1007/s00432-013-1421-5 (2013).

30 Swierczewska, M. et al. PTPRK Expression Is Downregulated in Drug Resistant Ovarian Cancer Cell Lines, and Especially in ALDH1A1 Positive CSCs-Like Populations. Int J Mol Sci 20, doi:10.3390/ijms20082053 (2019).

31 Chen, Y. W. et al. Receptor-type tyrosine-protein phosphatase kappa directly targets STAT3 activation for tumor suppression in nasal NK/T-cell lymphoma. Blood 125, 1589-1600, doi:10.1182/blood-2014-07-588970 (2015).

32 Ross, M. E. et al. Classification of pediatric acute lymphoblastic leukemia by gene expression profiling. Blood 102, 2951-2959, doi:10.1182/blood-2003-010338 (2003).

33 Yeoh, E. J. et al. Classification, subtype discovery, and prediction of outcome in pediatric acute lymphoblastic leukemia by gene expression profiling. Cancer cell 1, 133-143 (2002). 
53134 Boudot, A. et al. Differential estrogen-regulation of CXCL12 chemokine receptors, CXCR4 and CXCR7, contributes to the growth effect of estrogens in breast cancer cells. PLoS ONE 6, e20898, doi:10.1371/journal.pone.0020898 (2011).

35 Levoye, A., Balabanian, K., Baleux, F., Bachelerie, F. \& Lagane, B. CXCR7 heterodimerizes with CXCR4 and regulates CXCL12-mediated G protein signaling. Blood 113, 6085-6093, doi:10.1182/blood-2008-12-196618 (2009).

$36 \mathrm{Li}, \mathrm{T}$. et al. The expression of CXCR4, CXCL12 and CXCR7 in malignant pleural mesothelioma. The Journal of pathology 223, 519-530, doi:10.1002/path.2829 (2011).

37 Wang, J. et al. The role of CXCR7/RDC1 as a chemokine receptor for CXCL12/SDF-1 in prostate cancer. The Journal of biological chemistry 283, 4283-4294, doi:10.1074/jbc.M707465200 (2008).

38 Yoshida, D., Nomura, R. \& Teramoto, A. Signalling pathway mediated by CXCR7, an alternative chemokine receptor for stromal-cell derived factor1alpha, in AtT20 mouse adrenocorticotrophic hormone-secreting pituitary adenoma cells. Journal of neuroendocrinology 21, 481-488, doi:10.1111/j.13652826.2009.01867.x (2009).

39 Zabel, B. A., Lewen, S., Berahovich, R. D., Jaen, J. C. \& Schall, T. J. The novel chemokine receptor CXCR7 regulates trans-endothelial migration of cancer cells. Mol Cancer 10, 73, doi:10.1186/1476-4598-10-73 (2011).

40 Melo, R. C. C. et al. CXCR7 is highly expressed in acute lymphoblastic leukemia and potentiates CXCR4 response to CXCL12. PLoS ONE 9, e85926, doi:10.1371/journal.pone.0085926 (2014).

41 Brownlie, R. J. \& Zamoyska, R. T cell receptor signalling networks: branched, diversified and bounded. Nature reviews. Immunology 13, 257-269, doi:10.1038/nri3403 (2013).

42 Nika, K. et al. Constitutively active Lck kinase in T cells drives antigen receptor signal transduction. Immunity 32, 766-777, doi:10.1016/j.immuni.2010.05.011 (2010).

43 Serafin, V. et al. Glucocorticoid resistance is reverted by LCK inhibition in pediatric T-cell acute lymphoblastic leukemia. Blood 130, 2750-2761, doi:10.1182/blood-2017-05-784603 (2017).

44 LaForgia, S. et al. Receptor protein-tyrosine phosphatase gamma is a candidate tumor suppressor gene at human chromosome region 3p21. Proceedings of the National Academy of Sciences of the United States of America 88, 5036-5040, doi:10.1073/pnas.88.11.5036 (1991). estrogenically regulated protein tyrosine phosphatase gamma (PTPgamma) in human breast cancer cell line MCF-7. Oncogene 23, 1256-1262, doi:10.1038/sj.onc.1207235 (2004).

46 Chatterton, Z. et al. Epigenetic deregulation in pediatric acute lymphoblastic leukemia. Epigenetics 9, 459-467, doi:10.4161/epi.27585 (2014).

47 Funderburk, S. F., Wang, Q. J. \& Yue, Z. The Beclin 1-VPS34 complex--at the crossroads of autophagy and beyond. Trends in cell biology 20, 355-362, doi:10.1016/j.tcb.2010.03.002 (2010).

48 Polak, R. et al. Autophagy inhibition as a potential future targeted therapy for 
58049 Taganov, K. D., Boldin, M. P., Chang, K. J. \& Baltimore, D. NF-kappaBdependent induction of microRNA miR-146, an inhibitor targeted to signaling proteins of innate immune responses. Proceedings of the National Academy of Sciences of the United States of America 103, 12481-12486, doi:10.1073/pnas.0605298103 (2006).

50 Zhang, H. et al. MicroRNA patterns associated with clinical prognostic parameters and CNS relapse prediction in pediatric acute leukemia. PLoS ONE 4, e7826, doi:10.1371/journal.pone.0007826 (2009).

51 Churchman, M. L. \& Mullighan, C. G. Ikaros: Exploiting and targeting the hematopoietic stem cell niche in B-progenitor acute lymphoblastic leukemia. Exp Hematol 46, 1-8, doi:10.1016/j.exphem.2016.11.002 (2017).

52 Davidsson, J. et al. Tiling resolution array comparative genomic hybridization, expression and methylation analyses of $\operatorname{dup}(1 \mathrm{q})$ in Burkitt lymphomas and pediatric high hyperdiploid acute lymphoblastic leukemias reveal clustered nearcentromeric breakpoints and overexpression of genes in 1q22-32.3. Human molecular genetics 16, 2215-2225, doi:10.1093/hmg/ddm173 (2007).

53 Sanchez-Cuaxospa, M. et al. Low expression of Toll-like receptors in peripheral blood mononuclear cells of pediatric patients with acute lymphoblastic leukemia. International journal of oncology 49, 675-681, doi:10.3892/ijo.2016.3569 (2016).

54 Corthals, S. L. et al. Differential immune effects mediated by Toll-like receptors stimulation in precursor B-cell acute lymphoblastic leukaemia. $\mathrm{Br} \mathrm{J}$ Haematol 132, 452-458, doi:10.1111/j.1365-2141.2005.05893.x (2006).

55 Dorantes-Acosta, E. et al. TLR stimulation of bone marrow lymphoid precursors from childhood acute leukemia modifies their differentiation potentials. Biomed Res Int 2013, 846724, doi:10.1155/2013/846724 (2013).

56 Bauer, E. et al. Cooperation of ETV6/RUNX1 and BCL2 enhances immunoglobulin production and accelerates glomerulonephritis in transgenic mice. Oncotarget 7, 12191-12205, doi:10.18632/oncotarget.7687 (2016).

57 Torrano, V., Procter, J., Cardus, P., Greaves, M. \& Ford, A. M. ETV6-RUNX1 promotes survival of early B lineage progenitor cells via a dysregulated erythropoietin receptor. Blood 118, 4910-4918, doi:10.1182/blood-2011-05354266 (2011).

58 Silverman, J. A., Reynolds, L. \& Deitcher, S. R. Pharmacokinetics and pharmacodynamics of vincristine sulfate liposome injection (VSLI) in adults with acute lymphoblastic leukemia. J Clin Pharmacol 53, 1139-1145, doi:10.1002/jcph.155 (2013).

59 Inthal, A. et al. Role of the erythropoietin receptor in ETV6/RUNX1-positive acute lymphoblastic leukemia. Clin Cancer Res 14, 7196-7204, doi:10.1158/1078-0432.CCR-07-5051 (2008).

60 Krause, G., Hassenruck, F. \& Hallek, M. Copanlisib for treatment of B-cell malignancies: the development of a PI3K inhibitor with considerable differences to idelalisib. Drug design, development and therapy 12, 2577-2590, doi:10.2147/DDDT.S142406 (2018).

61 Liu, N. et al. BAY 80-6946 is a highly selective intravenous PI3K inhibitor with potent p110alpha and p110delta activities in tumor cell lines and xenograft models. Molecular cancer therapeutics 12, 2319-2330, doi:10.1158/15357163.MCT-12-0993-T (2013). 
62862 Hiebert, S. W. et al. The $\mathrm{t}(12 ; 21)$ translocation converts AML-1B from an 629 activator to a repressor of transcription. Molecular and cellular biology 16, $6301349-1355$ (1996).

63163 Papaemmanuil, E. et al. RAG-mediated recombination is the predominant driver 632 of oncogenic rearrangement in ETV6-RUNX1 acute lymphoblastic leukemia. Nature genetics 46, 116-125, doi:10.1038/ng.2874 (2014).

64 Montano, A., Forero-Castro, M., Hernandez-Rivas, J. M., Garcia-Tunon, I. \& Benito, R. Targeted genome editing in acute lymphoblastic leukemia: a review. BMC biotechnology 18, 45, doi:10.1186/s12896-018-0455-9 (2018).

65 Culbertson, M. R. \& Leeds, P. F. Looking at mRNA decay pathways through the window of molecular evolution. Current opinion in genetics \& development 13, 207-214, doi:10.1016/s0959-437x(03)00014-5 (2003).

66 Fuka, G., Kauer, M., Kofler, R., Haas, O. A. \& Panzer-Grumayer, R. The leukemia-specific fusion gene ETV6/RUNX1 perturbs distinct key biological functions primarily by gene repression. PLoS ONE 6, e26348, doi:10.1371/journal.pone.0026348 (2011).

67 Genitsari, S. et al. Biological Features of Bone Marrow Mesenchymal Stromal Cells in Childhood Acute Lymphoblastic Leukemia. Turkish journal of haematology : official journal of Turkish Society of Haematology 35, 19-26, doi:10.4274/tjh.2017.0209 (2018).

68 Nwabo Kamdje, A. H. et al. Mesenchymal stromal cells' role in tumor microenvironment: involvement of signaling pathways. Cancer biology \& medicine 14, 129-141, doi:10.20892/j.issn.2095-3941.2016.0033 (2017).

69 Bonilla, X., Vanegas, N. P. \& Vernot, J. P. Acute Leukemia Induces Senescence and Impaired Osteogenic Differentiation in Mesenchymal Stem Cells Endowing Leukemic Cells with Functional Advantages. Stem cells international 2019, 3864948, doi:10.1155/2019/3864948 (2019).

655

656 
660 Figure 1. E/R expression levels by RT-qPCR. Control clones showed an expression of E/R similar to it

661 was observed in the parental REH cells. In E/R KO clones, whose sequence was edited by the

662 CRISPR/Cas9 system, KO2 and KO3 showed a total loss of E/R expression and KO1 showed a leaky

663 expression. All the experiments were carried out by triplicate, the means with the standard deviations for

664 each clone were represented. ${ }^{* * * P} P 0.001$ (unpaired $t$-test).

665

666 Figure 2. Transcriptomic analysis of E/R KO clones. Heat map of TOP50 differentially expressed

667 genes in E/R KO clones as compared with REH cells and control clones. Each row represents one

668 differentially expressed gene; each column represents one clone. The dendrogram on the top reveals the

669 sample clustering; the dendrogram on the left reveals the gene clustering.

670

671 Figure 3. In vitro functional studies after $\mathbf{E} / \mathbf{R}$ abrogation. (A) Cell cycle distribution of control clones 672 and E/R KO cells at $48 \mathrm{~h}$. (B) CFSE quantification by flow cytometry after 48 in culture. The peak on the 673 right $\left(10^{3}\right)$ represents the percentage of cells that have not divided and the left peak $\left(10^{2}\right)$ represents the 674 percentage of cells that have divided and therefore diluted their CFSE expression. (C) CFSE expression 675 by flow cytometry of cells co-cultured with MSC cell line HS-5 at 48h. (D) Apoptosis level quantification

676 by PI expression. The figure shows the percentage of PI negative cells (left) and PI positive cells (right) at $67748 \mathrm{~h}$. (E) Apoptosis level quantification by PI expression after treatment with Vincristine $(1 \mu \mathrm{M})$ at $48 \mathrm{~h}$. 678 On the right is represented the mean distribution of control clones (dark grey) and E/R KO clones (grey) 679 of different experiments. All the experiments were carried out by triplicate. ${ }^{*} P \unlhd 0.05$ (unpaired $t$-test).

680

681 Figure 4. Western blot analysis of E/R targets expression. Lower phospho-Akt (60 kDa), BCL-XL (30

$682 \mathrm{kDa})$ and BCL-2 (28 kDa) expression levels were observed in all E/R KO clones compared with parental 683 cell line (REH) and control clones.

685 Figure 5. Cell viability and protein expression measured after Copanlisib / Prednisolone treatment.

686 (A) Cell viability was measured by the MTT proliferation assay after treatment (192 h) with Copanlisib 687 (10 nM). E/R KO clones (light grey square line) showed a higher sensitivity to Copanlisib than REH cells 
688 (black square line) and control clones (grey square line). This graph represents the average of three

689 independent experiments and in turn the average of the 2 control clones and the $3 \mathrm{KO}$ clones. (B) p-Akt

$690(60 \mathrm{kDa})$ and $\mathrm{p}-\mathrm{mTOR}(289 \mathrm{kDa})$ expression levels decreased after treatment with Copanlisib. (C)

691 Prednisolone (black square line), Copanlisib (dark grey square line) and Copanlisib plus Prednisolone

692 combination (grey square line) were tested in the different clones. The relative cell viability was

693 calculated as the percentage of untreated cells. $* P \unlhd 0.05 ; * * P \leq 0.005$ (unpaired $t$-test).

695 Figure 6. In vivo effects of CRISPR-mediated editing of the $\boldsymbol{E} / \boldsymbol{R}$ oncogene. (A) External appearance

696 of mice and developed tumours 48 - 62 days after subcutaneous cell injection. Tumours formed by KO

697 clones (right flank) were smaller than those induced by REH cells or control clones (left flank). (B)

698 Evolution of tumour growth measured every 2-3 days until the moment in which mice were sacrificed.

699 (C) Representation of the mean tumour size corresponding to each clone, independently of the group.

$700 * P \unlhd 0.05 ; * * \triangle 0.005$ (unpaired $t$-test). 


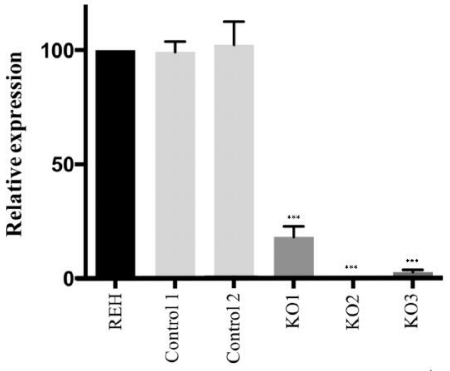


E/R KO clones vs control clones - Top 50 genes

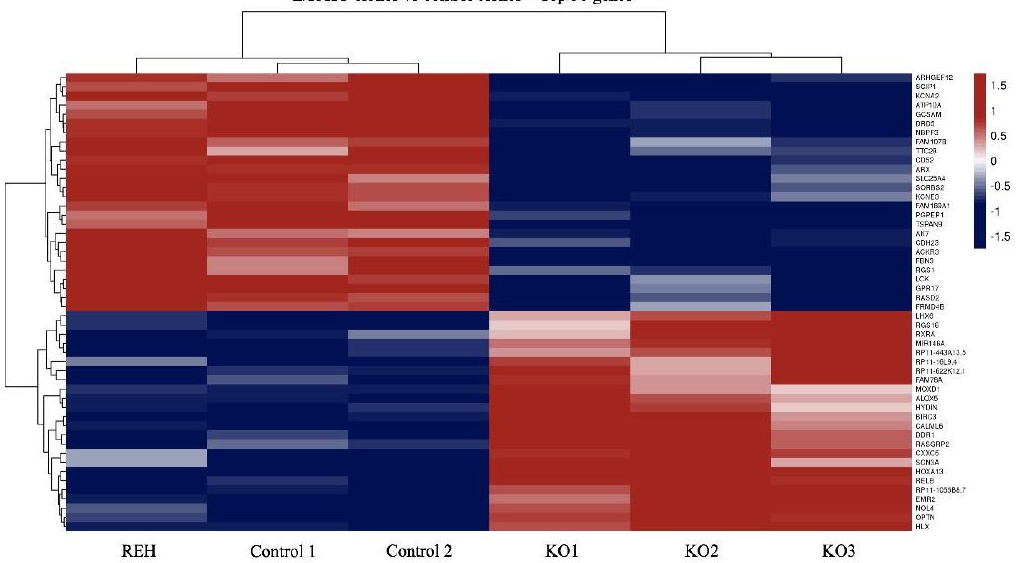


Bcl-2

$\beta$-actin

Bcl-xL

a//3-Tubulin

p-Akt

$\beta$-actin
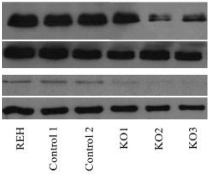
A

Copantistb $10 \mathrm{nM}$

90

B0

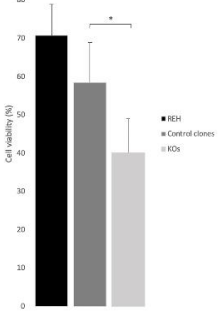

B

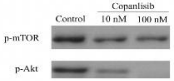

B-action

C.

Predaisolone 25b pM+Copunlisib 14nR

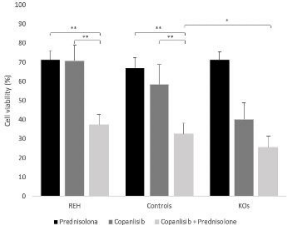


A
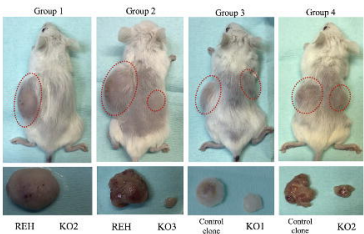

Cousel KOI Coubel
sloac
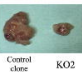

B

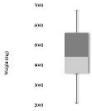

$\operatorname{sen}$

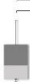

C

7000

0000

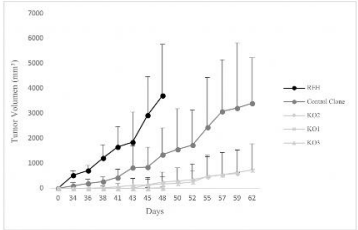

14:

Coraten
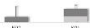

aw

wes

C 